\title{
The Basophil Activation Test for Clinical Management of Food Allergies: Recent Advances and Future Directions
}

\author{
Daniela Briceno \\ Noriega' \\ Malgorzata Teodorowicz' \\ Huub Savelkoul' \\ Janneke Ruinemans-Koerts $\mathbb{D}^{1,2}$ \\ 'Cell Biology and Immunology Group, \\ Wageningen University \& Research, \\ Wageningen, the Netherlands; \\ ${ }^{2}$ Laboratory of Clinical Chemistry and \\ Hematology, Rijnstate Hospital, Arnhem, \\ the Netherlands
}

\begin{abstract}
The basophil activation test (BAT) is an ex vivo functional assay that measures by flow cytometry the degree of basophil degranulation after stimulation with an allergen. In recent years, there has been an increased interest in the diagnostic value of the BAT as it has the potential to mimic the clinical phenotype of sIgE sensitized patients, in contrast to allergen-specific IgE levels. This diagnostic potential would be of particular interest for food allergies present early in life such as peanut, cow's milk and eggs, which require an expensive, time-consuming and patient unfriendly oral food challenge (OFC) for diagnosis. However, routine applications of the BAT for clinical use are not yet feasible due to the lack of standardized protocols and large clinical validation studies. This review will summarize the current data regarding the application of the BAT in food allergy (FA) for cow's milk, egg and peanut, being the most common causes of FA in children. Additionally, it will discuss the hurdles for widespread clinical use of the BAT and possible future directions for this diagnostic procedure.
\end{abstract}

Keywords: basophil activation test, food allergy, diagnosis, peanut, cow's milk, chicken egg

\section{Introduction}

Most food allergies (FA) are diagnosed in early life, nevertheless the diagnosis can be made in older children or even adults. Currently there are fourteen foods listed as recognized food allergens in the European Union, ${ }^{1}$ of which eight are responsible for approximately $95 \%$ of FA cases: cow's milk, eggs, fish, peanuts, shellfish, soy, tree nuts and wheat. ${ }^{2}$ In the US, approximately $9 \%$ of adults have a FA diagnosis; ${ }^{3}$ meanwhile, in Europe it stands at about $6 \%$ among adults. ${ }^{4}$ In children below the age of five the prevalence of FA ranges between $4 \%$ and $10 \%$ worldwide. 5,6

An oral food challenge (OFC) is the current gold-standard for FA diagnosis; however this method is labor intensive, costly and implies the risk of a severe and life threatening anaphylaxis. Determination of the prevalence of food allergies is dependent on confirmation by this gold standard; however, since OFCs are not only time-consuming but carry an inherent risk they might not be acceptable to all study participants. $^{7}$

FA varies in etiology, allergen type and severity which contributes to a challenging diagnosis. Adding complexity to this diagnosis there is also the cross-reactivity between allergens, the concomitant presence of multiple food allergens and food intolerances. ${ }^{8}$ The symptoms of a FA and a food intolerance can overlap, therefore
Correspondence: Janneke RuinemansKoerts

Cell Biology and Immunology Group, Wageningen University \& Research, Wageningen, the Netherlands

Email j.ruinemans-koerts@rijnstate.nl 
Resting Basophil

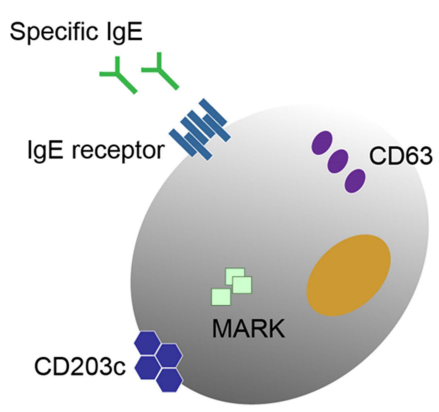

Activated Basophil

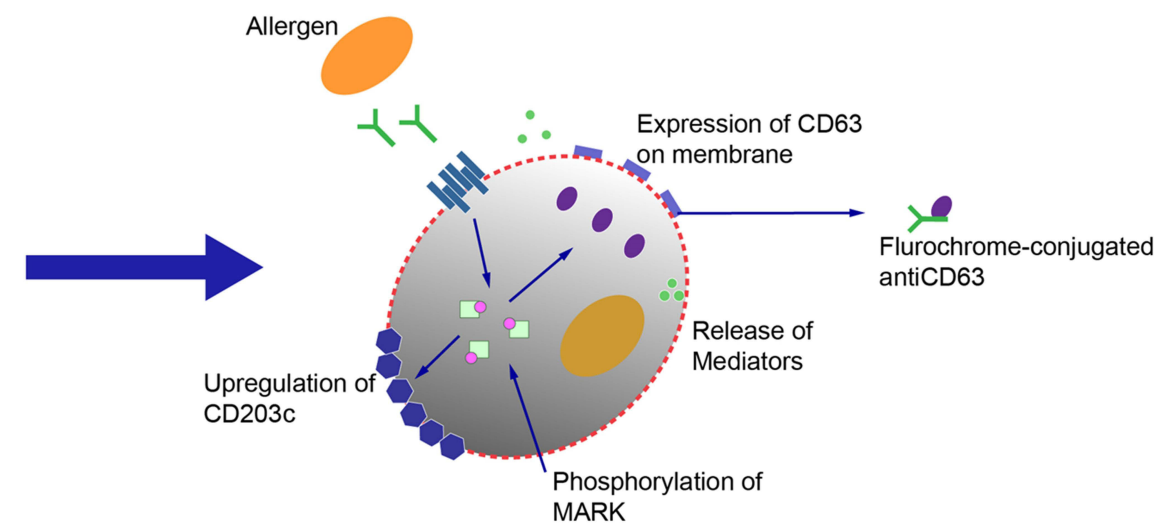

Figure I Basophil activation test principle. At a resting mode, the activation marker CD203c is expressed at low levels, but upon activation it is rapidly up-regulated. In addition, when the basophil is in a resting mode the activation marker CD63 is mainly present inside the cell granules. Upon activation, after exposure to an allergen, the granules fuse with the cell membrane and CD63 is exposed on the cell surface and can be detected by labelled antibodies with subsequent flow cytometry. Therefore the expression of CD63 is closely associated with degranulation.

a proper differential diagnosis is crucial since a FA triggers an immune response which can end in a life-threatening event known as anaphylaxis. The current cornerstone of FA diagnosis is the clinical history aided in most cases by specific IgE levels in blood (sIgE) and/or skin prick test (SPT). Both tests have a high sensitivity but low specificity; both detect $\operatorname{sigE}$ sensitization which is not the same as a food allergy; in other words sensitization does not necessarily lead to the development of symptoms. Additionally, both assays currently lack reliable threshold values as they are dependent on factors like the type of allergen/food and study population (eg, age, other diseases). The next step for food allergy diagnosis is an OFC; particularly the doubleblind placebo-controlled food challenge (DBPCFC). Because of the drawbacks of these diagnostic challenges, FA diagnosis is trending towards the development of screening tests reducing the indications for OFCs. This approach, however, requires the establishment of thresholds values for SPT and sIgE that can predict the likelihood of a clinical reaction. ${ }^{9}$ The burden of a FA misdiagnosis manifests in increased anxiety and diminished quality of life for patients plus potential nutritional deficiencies due to dietary restrictions. ${ }^{10}$ Furthermore, the economic cost of FA misdiagnosis to society are not insignificant with many resources being dedicated to further medical evaluation, additional testing and unnecessary prescriptions. ${ }^{11}$

Under this scenario, the interest in the basophil activation test (BAT) has increased as a potential tool which simulates an allergic reaction ex vivo. The BAT is a flow cytometric assay that detects the functional ability of $\operatorname{IgE}$ to activate basophils which are stimulated due to allergen exposure (see Figure 1). However, there is still knowledge lacking regarding degranulation metrics and implementation guidelines to guarantee universality in the execution of the test and interpretation of the results. ${ }^{12,13}$ The BAT measures the expression of activation markers (mainly CD63 or CD203c) on the basophil cell membrane following cross-linking of IgE antibodies caused by an allergen. ${ }^{14,15}$ Even though basophils are scarce (they form less than $3 \%$ of peripheral white blood cells) they are easily accessible cells and their activation is quantifiable. Therefore, the BAT is a promising biomarker for the diagnosis of a food allergy and can replace the OFC.

The BAT has been validated for different IgE-mediated food allergies and showed a high sensitivity and specificity. ${ }^{16,17}$ The accuracy of the BAT has been shown to be higher than tests for IgE sensitization (sIgE/SPT) ${ }^{12,17}$ and the BAT has been able to differentiate clinically allergic patients from those who were sensitized but tolerant, with a specificity ranging from $75 \%$ to $100 \%$ and a sensitivity between $77 \%$ to $98 \%{ }^{12,15-17}$ This specificity and sensitivity profile has positioned the BAT as a potential tool in reducing the number of $\mathrm{OFCs}^{12}$ contributing to the interest in further developing this technique for FA diagnosis.

One reason for this improved diagnostic power can be attributed to the fact that the BAT is a functional assay, therefore the results are not dependent on the amount of $\operatorname{IgE}$ alone but also on its characteristics such as affinity, avidity and even on antibodies of other isotypes (eg, IgG4). ${ }^{18}$ 
Kits for performing the BAT have become more readily available for any clinical or research laboratory with a flow cytometry facility. ${ }^{19}$ Nonetheless, standardization and harmonization of the BAT technique plus interpretation of results are still lacking and not yet defined. Many questions remain regarding how feasible it is to standardize the BAT and its methodology while adopting a protocol that allows for comparison of results between different centers. Simultaneously, universal threshold values should be established and data for specific allergens validated in different allergic populations whilst evaluating the cost-effectiveness of this technique. It is important to keep in mind when validating the BAT in the context of cross-reactivity syndromes that the performance of the test will be influenced by the control group, a healthy control group will likely overestimate the specificity of the test and therefore also the diagnostic accuracy of the reference test. $^{13}$

\section{The BAT and Food Allergy Diagnosis}

Food allergies that begin at an early age such as cow's milk, egg and peanut, present a higher diagnostic challenge as performing an OFC in children generates significant anxiety in parents and clinical staff. Furthermore an OFC in young children can be difficult to perform (eg, willingness of the patient to eat) and interpret since the majority of the symptoms are subjective and the test is often not continued until objective symptoms are reached. $^{20,21}$ Both milk and egg allergies start early in life and the majority becomes naturally tolerant over time whereas peanut allergy starts later in life and is usually lifelong. Therefore, in this subgroup of FA, the BAT as a tool would represent a significant diagnostic improvement in FA diagnosis in the clinical practice as it has the potential to reduce the need for an OFC. ${ }^{22}$ Furthermore, most of the clinical validation data currently available for the BAT are obtained with egg, milk and peanut; therefore, these data are used to deduce and sum up the current clinical application of the BAT. Although most articles regarding the BAT focus on diagnosis there is pertinent data available regarding the role of the BAT in monitoring the effect of immunotherapy as well. ${ }^{23}$

The BAT mimics the clinical phenotype of patients while other allergy tests can only detect the presence of allergen-specific IgE. Additionally, the BAT can differentiate sensitization from a true food allergy and thus segregate between allergy and tolerance, particularly in children with peanut or egg allergy. ${ }^{24}$

\section{Peanut Allergy}

Peanut allergy (PA) is one of the most common food allergies worldwide accounting for $0.5 \%$ to $1.5 \%$ of the population in Western countries. ${ }^{25}$ PA usually starts around 18 months of age but can begin later in life, either as stand-alone allergy or as part of the pollen-food allergy syndrome. ${ }^{26} \mathrm{PA}$, along with tree nut allergies, is the most common cause for life threatening anaphylactic reactions. Even though only a small percentage of US children are clinically allergic to peanut (currently 1.4\%), approximately $10 \%$ are sensitized to it; consequently, the proper diagnosis in this group is vital for the quality of life of the patients and their families. ${ }^{27}$ In FA diagnosis, it is becoming critical to be able to identify the individual disease phenotype to provide the correct individualized treatment; thus, techniques that can separate cross-reactivity with other allergen families and pollen allergen are gaining importance ${ }^{28}$ For example; Arachis hypogea 2 (Ara h 2) specific IgE (reflecting sensitization) has been shown to be a useful predictor of clinical reactivity. ${ }^{29}$ In children with suspected PA, the BAT diagnosed PA with $98 \%$ specificity and $75 \%$ sensitivity. Moreover, the BAT was the best biomarker for severity, identifying severe reactions with 97\% specificity and $100 \%$ sensitivity. ${ }^{30}$ Thus, the BAT outperformed the level Ara h 2 specific-IgE, level of peanut specific-IgE and $\operatorname{IgG} 4 / \operatorname{IgE}$ ratio, only to be surpassed by the SPT. ${ }^{30}$ Therefore, the BAT is capable of predicting the allergic clinical status to peanut in children and could reduce the need for OFCs. ${ }^{12,30}$

It has been observed that a higher proportion of activated basophils is associated with more severe reactions and a lower threshold of activation, raising the question if severity is linked to threshold. For example; in adults that suffer from severe peanut allergy similar associations have been identified using the BAT, where basophil reactivity to peanut was significantly higher in patients who had a history of severe allergy to peanuts when compared to patients who were sensitized to peanuts $(p<0.001) .{ }^{31}$ However, further validation is necessary before applying this result more broadly including to other food allergens and to other patient populations. ${ }^{28}$

Moreover, single peanut or tree-nut allergic patients are often sensitized to other tree-nuts requiring multiple OFC for diagnosis since the main challenge with these patients is distinguishing between sensitization and allergy. The 
BAT has shown to be capable to discriminate between allergic and nonallergic children, to the respective nut/ seed when tested against peanut, hazelnut, cashew nut, sesame, and almond. ${ }^{32}$ Moreover, this study reported that the use of the BAT as a second step in the diagnostic process reduced the number of OFC by $5 \%$ to $15 \%$ after equivocal SPT and IgE to extracts and components. This notion was later reinforced by further studies showing that separately the SPT and the BAT were limited in their capacity to distinguish allergy from tolerance; however, when used in conjunction as part of the diagnostic kit their ability to identify allergic and tolerant patients improved. ${ }^{33}$ Moreover, the same study also concluded that this approach could potentially reduce the need for OFCs with $78.2 \%$ in walnut/pecan cases and with $76.6 \%$ in cashew/pistachio cases. ${ }^{33}$ Additionally, the BAT has been useful to differentiate between the allergenicity of different allergen extracts in hazelnut allergic patients. In 132 hazelnut allergic patients, sensitization was confirmed by SPT and sIgE against hazelnut; ${ }^{34}$ following a hazelnut free diet, a DBPCFC was performed with increasing amounts of native and roasted hazelnut. The BAT was measured before and after provocation and it showed that significantly higher concentrations of the allergen extract were needed (roasted $>$ native) to induce $50 \%$ basophil activation. Therefore, the researchers concluded that the BAT was useful in determining the reactivity of an allergen extract. $^{34}$

In PA patients, oral immunotherapy (OIT) can significantly shift the threshold dose of peanut that can be ingested without generating symptoms. Sustained protection during and after OIT has been reported in association with lower levels of basophil activation at 13 weeks after active OIT. ${ }^{35}$ Hence, patients with a low basophil responsiveness after OIT were more likely to achieve treatment success. ${ }^{36}$ Using the Ara h 2 as a predictor of clinical reactivity, the BAT could provide a functional surrogate of efficacy since studies have demonstrated that basophil sensitivity to Ara h 2 is a useful biomarker for long time efficacy of peanut OIT. ${ }^{37}$ Therefore there is increasing data that the BAT provides additional value in monitoring the response to immunotherapy. ${ }^{38}$

\section{Cow's Milk Allergy}

Cow's milk allergy (CMA) is the most common childhood allergy with a prevalence of approximately $2.5 \%$ worldwide. ${ }^{20}$ The onset of this allergy is generally related to the introduction of cow's milk based infant formula and currently it affects 1 in every 50 infants under the age of one with most of the patients outgrowing their allergy during childhood or pre-puberty. ${ }^{39} \mathrm{CMA}$ is highly variable with allergens involved in the allergic response. Previously, ß-lactoglobulin, the most abundant whey protein, was thought to be the most important allergen in CMA since it is not present in human milk and up to $76 \%$ of the patients react to this protein. ${ }^{40}$ Nowadays, it is known that other proteins, including $\alpha$-lactalbumin and caseins, are critically involved in the disease. Low levels of ß-lactoglobulin and casein allergen-specific (known as Bos d 8) sIgE concentrations have been described as predictive for the resolution of CMA. ${ }^{41}$

The lack of specific clinical manifestations can often lead to a misdiagnosis; the current diagnostic tests include sIgE (sensitivity $87 \%$, specificity $48 \%$ ) and SPT (sensitivity $88 \%$, specificity $68 \%$ ) while the current gold standard for CMA diagnosis is the double-blind placebo-control food challenge (DBPCFC) ${ }^{41,42}$ The difficult diagnostic scenario has resulted in an over-diagnosis of CMA, possibly undermining breast feeding rates and leading to unnecessary elimination diets with negative nutritional impact. ${ }^{43}$ For example, a meta-analysis showed that the self-reported prevalence of CMA was approximately $6 \%$, however the prevalence fell to $0.6 \%$ when CMA was confirmed by DBPCFC. ${ }^{41}$ The BAT has been described as highly efficient in confirming CMA in children ${ }^{44}$ with a sensitivity of $89 \%$ and a specificity of $83 \%$ for cow's milk extract, ${ }^{45}$ positive predictive value of $81 \%$ and negative predictive value of $96 \%$ in detecting persistently allergic patients. ${ }^{46}$ For safety and commercial reasons, milk is processed by using various treatments (heat and other physio-chemical) that can alter the allergenic potency of milk proteins. The BAT can help to distinguish between patients that can tolerate heated forms of cow's milk from those who are not able to. Therefore, the BAT can provide important information which has implications for the prognosis of CMA patients as patients who persistently do not tolerate heated milk will have a higher chance to develop a more persistent form of CMA allergy. ${ }^{47,48}$

Even though most patients diagnosed with CMA will outgrow this allergy in the first years of life, the prognosis for those who do not is worse; with cow milk IgE levels reported being highly predictive for this outcome. ${ }^{20}$

Therefore, in FA that are commonly outgrown with time such as CMA, the BAT can be useful in assessing the natural resolution of food allergies and in deciding when to reintroduce cow's milk to the patient's diet. The 
BAT has been described as highly effective in improving the diagnostic accuracy in CMA since it can mimic the acute degree of CMA against cow's milk and human milk allergens as well as assist in monitoring the development of CMA $;{ }^{49}$ offering the possibility that the BAT could be a reliable and cost effective diagnostic tool when clinicians suspect an IgE mediated CMA which could in turn diminish the need for DBPCFCs. ${ }^{50}$

In recent years, many studies have demonstrated the efficacy of OIT for CMA ranging from $67 \%$ tolerance at 18 weeks $^{51}$ to $90 \%$ showing complete desensitization after 1 year. $^{52}$ Nevertheless, follow-up studies have found that full milk tolerance decreases dramatically over time, which suggests that protection is more difficult to maintain than previously described. ${ }^{53}$ Current recommendations suggest treatment of CMA should wait until the child is 3 years old; however, recent findings suggest that OIT is a promising strategy for CMA even in young children. OIT has demonstrated to achieve full tolerance in a high percentage of children with mild side effects that can be easily managed by slowing the desensitization. ${ }^{54}$ At the end of oral food allergy desensitization, a significant decrease in specific IgE levels and increase in specific IgG4 levels is described in the literature, highly possible due to a switch from a Th2 to a Th1 response, ${ }^{55}$ therefore by analyzing the up-regulation of allergen-induced CD63 with flow cytometry it is possible to monitor the progression of clinical tolerance by OIT in FA. There is little information regarding the use of the BAT for monitoring the clinical tolerance induced by OIT in CMA; however, a reduction of cow's milk protein-induced CD63 expression levels at the end of the desensitization protocol has been observed and the BAT has been described as highly sensitive and closely correlated with clinical tolerance. ${ }^{56}$

\section{Egg Allergy}

Egg allergies are common IgE mediated food allergies in children with a prevalence ranging from $1.3 \%$ to $10.1 \%,{ }^{20}$ with most cases presenting during the first year of life. The prognosis is generally good with the majority of children outgrowing their allergy at school age. A proper diagnosis of egg allergy is crucial for several reasons, which can also be valid for other food allergies, (1) it can cause severe allergic reactions in sensitized children, (2) unnecessary avoidance due to a misdiagnosis leads to significant dietary restrictions and the possibility of nutritional deficiencies, (3) early sensitization to egg is a known marker of later sensitization to aeroallergens and the possible future development of asthma and (4) many vaccines that are administered during childhood contain egg therefore an egg allergy diagnosis is crucial to determine which vaccines are safe to administer. ${ }^{57}$

Most of the allergenic proteins are found in the egg white: ovomucoid (Gal d 1), ovalbumin ( Gal d 2), ovotransferrin (Gal d 3), egg white lysozyme (Gal d 4) and ovomucin; with Gal d 2 ovalbumin being the most abundant protein in egg white. ${ }^{58}$ Currently the BAT for egg allergy is described with a sensitivity of $63 \%$ and a specificity of $96 \%$ for CD203c expression and a sensitivity of $77 \%$ and a specificity of $100 \%$ for CD63 expression using ovalbumin. ${ }^{17}$ An important diagnostic conundrum for clinicians is the distinction between allergic and sensitized children (clinically tolerant but demonstrate a positive sIgE and/or SPT). Studies have attempted to evaluate if the BAT can play a role in addressing this diagnostic problem, which when presented requires further evaluation with an OFC. At the moment, the data suggests that most egg-sensitized but tolerant children are unable to elicit basophil activation upon allergen challenge. ${ }^{59}$ Therefore, the BAT might be a handy tool to complement conventional tests in this group of patients allowing for a better differentiation between allergic and non-allergic patients. $^{45}$

Generally, individuals with egg allergy can tolerate cooked products that contain egg which would imply that the allergic response is dependent on epitope configuration. The ovalbumin proteins are heat labile while the ovomucoid epitopes do not seem to be affected by heating. Some heat labile allergenic proteins change their configuration during the cooking process and therefore their immunogenic potential is either blunted or minimized. ${ }^{60}$ Alternatively, partially unfolded proteins could expose existing hidden epitopes and increase their allergenic activity. So, net effects are hard to predict without preexisting knowledge or careful analysis of epitopes present in the allergens and severe allergic reactions can occur with a single bite of cooked egg (about 70mg of egg protein). Patients that are diagnosed with an egg allergy are placed on egg-free diets, but total egg avoidance is very challenging both for patients and caregivers. Therefore, new treatment strategies are being explored. Food oral immunotherapy can induce tolerance or desensitize patients that are allergic to egg and it is associated with a median success rate of over $80 \%{ }^{61}$ The aim of the OIT in the treatment for a FA, as previously indicated, is to achieve tolerance to the usual or certain doses of the 
allergenic food to prevent reactions in case of ingestion of small quantities of said food. This is mainly attributed to several immunological responses, mainly a decrease in specific IgE, increase in specific IgG4 antibodies, formation of specific regulatory $\mathrm{T}$ cells and changes in the basophil response. $^{62}$

Research has shown that in OIT in egg allergic children produces a reduction in basophil activation after allergen stimulation; a significant decrease in percentage of CD203c + cells $(\mathrm{p}=0.04)$ and a lower percentage of CD63+ cells $(\mathrm{p}$ $=0.07$ ) over time after stimulation with $0.01 \mu \mathrm{g} / \mathrm{mL}$ antiovomucoid. ${ }^{63}$ This could be caused by changes in circulating levels and surface bound $\operatorname{IgE}$ on the basophils and in the amount of $\operatorname{IgE}$ receptors on the basophil surface. Therefore, using the BAT to test the safety and efficacy of a new immunotherapy such as low allergenic hydrolyzed egg is a handy tool for researchers and clinicians to circumvent expensive OFCs.

\section{Future Directions of the Basophil Activation Test}

The BAT is currently not widely used in FA diagnosis, although it possess a high diagnostic potential, it lacks validation and standardization to allow results comparisons between different laboratories and protocols that can be universally applied need to be formulated. This process should include not only the proper reference test that would be used to validate and standardize the BAT, but also the considerations of the possible drawbacks of utilizing such a reference and additionally a protocol for the allergens used and their importance in each subgroup of patients, as well as which activation markers are better suited for a specific patient population. These are among the main factors that need to be clearly established before the BAT can be universally used for clinical diagnosis or research (see Table 1).

\section{BAT Validation and Standardization, the Reference Test}

Standardization and validation of a new diagnostic test starts with the choice of the reference test(s). Currently, OFCs outcomes are used as the gold standard for FA diagnosis. A DBPCFC would be the best test as it removes patient and observer bias; ${ }^{64}$ therefore, it should be the reference test for validation and standardization of a newer diagnostic tool such as the BAT. However, even this gold standard has limitations as 3\% false-positive, $3 \%$ false-negative results and $10 \%$ dubious outcomes occur. ${ }^{65-}$

${ }^{67}$ In addition, there are several other points of concern like differences in dosing scheme and type of food matrices. Uniformity concerning these issues has not been obtained yet. Furthermore, reproducibility of the OFC has not been extensively tested. However, Glaumann et al found that that the threshold of the OFC showed a much higher variation and lower reproducibility than the sensitivity of the BAT (CD-sens). ${ }^{68}$ Finally, although there are guidelines for the stop criteria of an OFC (ie, Practall ${ }^{69}$ ) the question remains whether subjective symptoms are similar to objective symptoms in predicting the degree of severity and avoidance of food allergen traces. Thus, even though the OFC is the best diagnostic tool currently available, uncertainties remain about its value in clinical practice. Therefore, when validating the BAT by using the OFC, the limitations of the reference test need to be considered. This also urges us to rethink the applications of the OFC and new diagnostic tools, with their own drawbacks, in the optimization of food allergic diagnostics. Nevertheless, like the gold standard, a new diagnostic tool should be extensively validated in robustness and applicability. For the BAT it means that much research is still required concerning the source and type of allergens used, the optimal readout-parameters and the determination of reliable and safe cut-off values.

\section{Allergen Selection}

The BAT is a functional assay that evaluates the activation state of basophil cells before and after stimulation with allergens. The structure and availability of IgE-binding epitopes of the allergens in the food is influenced by food matrix composition as well as food processing steps before exposure to the patient. For food diagnostic purposes, raw allergen preparations are often used while individuals are mostly exposed to processed foods, eg in the case of soy or peanut proteins. Therefore, the use of raw extracts can compromise the diagnostic procedure and interpretation of the efficacy of tolerance induction; the BAT gives the possibility to test various allergen preparations which gives it a diagnostic advantage over other techniques, like the OFC.

The expected response in an allergic patient in a BAT test is a bell shape dose-response curve. Nonetheless the complexity of antigens and the affinity of different profiles of epitope-specific IgE can vary the shape of the doseresponse curve. ${ }^{70}$ Several factors have an impact on the basophil surface activation markers dose-response curve, 
Table I Technical Considerations for Widespread Use of the BAT

\begin{tabular}{|c|c|}
\hline \multicolumn{2}{|c|}{ Validation and Standardization } \\
\hline $\begin{array}{l}\text { Current } \\
\text { Challenge }\end{array}$ & How to use the proper test to validate and standardize a new tool such as the BAT \\
\hline Proposal & $\begin{array}{l}\text { DBPCFC best current option } \\
\text { - Gold standard for FA diagnosis } \\
\text { - Removes patient and observer bias }\end{array}$ \\
\hline Pitfalls & $\begin{array}{l}\text { DBPCFC has limitations } \\
-3 \% \text { false positives, } 3 \% \text { false negatives and } 10 \% \text { dubious outcomes }\end{array}$ \\
\hline \multicolumn{2}{|c|}{ Allergen Preparation } \\
\hline $\begin{array}{l}\text { Current } \\
\text { Challenge }\end{array}$ & $\begin{array}{l}\text { Factors impact the basophil surface activation markers dose-response curve: affinity of the antigen for the cell-bound lgE } \\
\text { antibodies, density of the epitope-specific lgE antibodies and epitope spreading of the } \lg E \text { antibody }\end{array}$ \\
\hline Proposal & Include a broad range of allergen concentrations to better evaluate the effect of the allergen in the basophil response \\
\hline Pitfalls & $\begin{array}{l}\text { Most FA diagnostic tools include mainly water-soluble allergens, thus lipophilic allergens are missing } \\
\text { Which processed allergens should be used in the BAT still require research }\end{array}$ \\
\hline \multicolumn{2}{|c|}{ Basophil Identification/Activation Markers } \\
\hline $\begin{array}{l}\text { Current } \\
\text { Challenge }\end{array}$ & $\begin{array}{l}\text { For a valid interpretation of the results, the precise identification of the basophil population is crucial } \\
\text { Since several markers can be measured, the BAT is a valuable tool as it can assess various immunological pathways providing } \\
\text { valuable insights into immune mechanisms of allergic disease }\end{array}$ \\
\hline Proposal & Studies that compare different identification and activation markers in the same population of patients are needed \\
\hline Pitfalls & $\begin{array}{l}\text { Researchers need to be aware that the current data suggests that depending on the allergen and the cohort used, there might be } \\
\text { variability in the sensitivity and specificity of the basophil activation markers. }\end{array}$ \\
\hline \multicolumn{2}{|c|}{ Read-out Parameters } \\
\hline $\begin{array}{l}\text { Current } \\
\text { Challenge }\end{array}$ & $\begin{array}{l}\text { Establish the most effective way to measure basophil activity. At the moment, it is advised to measure to first measure basophil } \\
\text { reactivity and then measure basophil sensitivity. }\end{array}$ \\
\hline Proposal & $\begin{array}{l}\text { The area under the dose-response curve (AUC), a marker of both reactivity and sensitivity is gaining attention since it measures } \\
\text { at multiple allergen concentrations lowering the risk of false outcomes }\end{array}$ \\
\hline Pitfalls & $\begin{array}{l}\text { "Basophil anergy", a non-responding basophil to lgE receptor-mediated signalling after stimulation with one or more types of } \\
\text { allergen remains a problem in approximately } 10 \% \text { of cases. }\end{array}$ \\
\hline
\end{tabular}

amongst them: affinity of the antigen for the cell-bound IgE antibodies, density of the epitope-specific $\operatorname{IgE}$ antibodies and epitope spreading of the IgE antibody. ${ }^{70}$ All of these factors determine the optimal allergen concentration for basophil activation and might vary significantly among subjects. To account for this issue it is advised to include a broad range of allergen concentrations to better evaluate the effect of the allergen in the basophil response.

Currently food allergy diagnostic tools mainly include water-soluble allergens and thus lipophilic allergens are missing. Using purified mixtures of Ara h 10/11 and Ara h $14 / 15$ (ie peanut lipophilic oleosins) the BAT showed that the rate of peanut oleosin sensitization among peanut allergic patients was $65 \% .^{71}$ This shows that sensitization to peanut oleosins is clinically relevant and therefore oleosins are major allergens in peanut allergy. Nevertheless, in order to evaluate the predictive value of these results they need to be compared with the outcome of the OFC and other peanut allergens with additional multicenter studies. $^{72}$

Peanut allergenic proteins are significantly affected by thermal processing which is caused by the interaction between the proteins and other food compounds such as sugars. An example of such interaction is the Maillard reaction, which involves the formation of neo-allergens by promoting the aggregation and formation of new epitopes. These neo-allergens may increase the severity of the allergic reaction in some individuals who are sensitized 
against processed and not raw allergens; therefore providing a need to include processed food in the diagnosis of FA, which normally use raw extract for PA diagnosis. Moreover, it is known that thermal processing affects the degranulation capacity differently, increasing in Ara $\mathrm{h} 1$ while decreasing in Ara $\mathrm{h} 2 / 6 ;{ }^{73}$ granting the BAT an important role in the assessment of food processing on protein allergenicity. As shown by Vissers et al, it is important to include degranulation assays in addition to the commonly studied IgE reactivity to have an inside in the clinical relevance of an allergen. ${ }^{73}$ The authors showed that the IgE-binding capacity of Ara h 1 roasted in the presence of sugar was decreased 9000 -fold compared with native Ara h 1, while the capacity to elicit mediator release was increased. In this area, the BAT has been described as helpful to determine the effect of thermal processing on the allergenicity of peanut proteins. A study which used both IgE immunoblotting and the BAT for raw and roasted peanuts did not find a correlation between the two techniques, highlighting the fact that IgE binding studies do not predict the potential of an allergen to trigger cell degranulation. The authors explained the discrepancy between a positive immunoblotting result and a negative BAT as an occurrence due to sensitization to heat-resistant crossreactive carbohydrate determinants, which is a main cause of positive IgE results that have no clinical significance. ${ }^{74}$ Consequently, the BAT provides important additional information to the results yielding from the $\operatorname{IgE}$ binding techniques.

In addition to providing valuable information regarding the allergenicity of peanuts and hazelnut, the BAT has yielded meaningful information about the non-specific lipid transfer proteins that are involved in allergies to fresh and processed fruits. The BAT showed that only severe heat treatment of Mal d 3 from apple peel caused significant decrease in its allergenicity, suggesting that the sugar in fruit may contribute to the thermostability of the allergenic activity ${ }^{75}$ Hence, the BAT is a suitable diagnostic tool to assess the allergenicity of processed allergens. Nonetheless, which processed allergens should be used in the BAT to reflect the in vivo situation still needs much research.

\section{Determination of the Degree of Basophil Activation \\ Activation Markers}

Following allergen stimulation, human basophils exhibit different degranulation patterns releasing various mediators and expressing particular activation markers. The BAT detects phenotype changes of allergen induced basophil degranulation; for this different protocols are in place using CCR3, CD123, CRTH2, CD203c or anti-IgE to identify basophils. All these markers are expressed on the basophil membrane but secondary makers are necessary to exclude CRTH2 + T cells or CD123+ plasmacytoid dendritic cells. Additionally, it has been suggested that $\mathrm{CD} 203 \mathrm{c}$ is the marker that reveals the purest basophil cell population when compared to other markers $\left(\mathrm{CCR}^{+}{ }^{+}, \mathrm{CRTH}^{+} / \mathrm{CD}^{-}, \mathrm{CCR}^{+} / \mathrm{CD}^{-}, \mathrm{IgE}^{+}\right.$, among others) and that anti-IgE should be used when a basophil population of very high purity is needed. ${ }^{76}$ For a valid interpretation of the results, the precise identification of the population of basophils is crucial. ${ }^{15}$ As long as these markers are not compared in one population it is difficult to determine which markers perform best; hence studies that compare different identification and activation markers in the same population are needed.

Moreover, degranulation is detected by surface expression of CD63, which is only expressed on the inner side of the granule membrane of the resting basophil. ${ }^{77}$ Since the development of the BAT, several activation markers have been studied and compared. In the mid 1990's the CD63 activation marker was discovered and currently is the most favored activation marker because it is directly related to histamine release and it is easily accessible since it is expressed in a distinct positive population. ${ }^{48,76,78}$ The other valuable marker is $\mathrm{CD} 203 \mathrm{c} ;{ }^{79}$ however, it does not form a distinct positive population and is a more general basophil marker which can be used both as an identification and as an activation marker. ${ }^{48}$ CD63 and CD203c are upregulated after IgE receptor aggregation but follow partially different metabolic pathways.

Presently, there is no standardization of CD63 or $\mathrm{CD} 203 \mathrm{c}$ detection and the determination of the positive threshold value. ${ }^{24}$ Furthermore, at several allergen concentrations, some studies have shown, that CD63 and CD203c expression differed between allergic or sensitized patients. ${ }^{76}$ For example, in hazelnut allergy CD203c expression showed a better discrimination capacity when compared to CD63 and therefore was more accurately able to distinguish between sensitized and allergic patients. ${ }^{79}$ This could be explained by the differences in kinetics between CD63 and CD203c. While the maximal upregulation for CD63 is between 25-30 minutes, for $\mathrm{CD} 203 \mathrm{c}$ it is at 10-20 minutes; in this study the 
stimulation time used averaged 15 minutes to capture both markers. ${ }^{79}$ In general, expression of CD203c reach their peak quickly and starts to decline after approximately 20 minutes and both CD63 and CD203c disappear after 4 to 5 hours of incubation.

A possible new marker is CD300, a surface receptor on basophils. This receptor was shown to increase during $\operatorname{IgE}$ mediated basophil degranulation but it is not necessarily elevated as a result of an allergen-mediated degranulation. The CD300 marker is correlated to enhanced degradation and its expression levels correlated with the severity of symptoms particularly in children with a severe CMA symptomatology; thereby suggesting that CD300c has a role in the clinical manifestation of CMA by decreasing the activation threshold of basophils. ${ }^{80}$ An additional marker of interest is CD300a, an inhibitory receptor that is rapidly up-regulated in response to IgE/FceRI (high affinity receptor for $\mathrm{IgE}$ ) and inhibits anaphylactic basophil degranulation. ${ }^{81}$ Furthermore, the expression of CD300a, similar to CD63, remained in a plateau for approximately 2 hours. CD300a is associated with inhibitory ITIMmediated signaling (immunoreceptor tyrosine-based inhibitory motifs) which may hamper activation processes. ${ }^{81}$ Moreover with an inhibitory net observed effect, several studies have reported a CD300a dominance over $\mathrm{CD} 300 \mathrm{c} ;{ }^{80,82}$ thus more precise tools are needed to characterize the functional input from CD300a and CD300c.

The current data suggest that depending on the allergen and the cohort used, there might be a variability in the sensitivity and specificity of the basophil activation markers. On the other hand, as several markers can be measured, the BAT is a valuable tool for researchers as it can assess various immunological pathways providing valuable insights into immune mechanisms of allergic diseases. ${ }^{22}$

\section{Result Analysis and Interpretation}

Since the analysis and interpretation of the results of the BAT can be intensive and laborious, recently a data driven programmatic approach has been proposed to analyze the flow cytometric results in a more reproducible, unbiased and productive way. By using the $\mathrm{R}$ Bioconductor package, flowCore, researchers were able to analyze 269 basophil activation tests from a clinical trial in a quick and efficient way, representing a net saving of 1340 minutes of labor by a skilled operator and only $2 \%$ of the basophil activation results differ significantly from manual gating. ${ }^{83}$ This data-driven approach could provide a platform for the BAT data to be analyzed in a more transparent and reproducible way with better quality control and additionally providing an adequate way for data sharing among clinicians and researchers.

Commonly there are two ways to measure basophil activity; by the number of basophil that respond to a stimulus (basophil reactivity) or by the allergen concentration at which half of all reactive basophils respond (basophil sensitivity); once reactivity is confirmed it may be useful to evaluate sensitivity. ${ }^{48}$ Basophil reactivity can be expressed as \%CD63+ basophils at a given allergen concentration and it refers to the proportion of basophils that express CD63 compared to the negative control. ${ }^{84}$ Basophil reactivity can also be expressed as the ratio of $\% \mathrm{CD} 63+$ to allergen $\mathrm{IgE}$ mediated positive control (anti-IgE or anti- FceRI) and recently two studies reported an association between basophil reactivity and symptom severity in PA patients. ${ }^{30,84}$ To measure sensitivity the reactivity at 6-8 allergen concentrations is measured, then the graded response is fitted to a curve of reactivity vs allergen concentration and the eliciting concentration at which $50 \%$ of basophils (EC50) is determined. $^{70}$ From this parameter, CD-sens can also be determined as $1 / \mathrm{EC}_{50} \times 100$. Basophil sensitivity has been reported to be helpful in the diagnosis of food allergy. ${ }^{12,70}$ Both basophil reactivity and sensitivity seem to be distinct parameters of activation; nonetheless both are regulated by Syk and appear to be interdependent. ${ }^{70}$

Recently, the area under the dose-response curve (AUC) is gaining interest since it is a marker of sensitivity and reactivity ${ }^{48,70}$ and it can be calculated in cases where responses do not fit well to a typical dose-response curve. $^{48}$ Furthermore, it uses several measuring points, at multiple allergen concentrations, which lowers the risk of false outcomes.

Therefore basophil reactivity and basophil sensitivity plus read-out parameter such as CD-sens, EC50 and AUC have been reported as sensitive biomarkers that reflect the clinical severity of anaphylactic reactions, the clinical thresholds for eliciting symptoms and OIT particularly in FA patients. ${ }^{85}$ A result of increased basophil reactivity and sensitivity reported in thresholds of BAT parameters, can help a clinician decide whether or not to perform an oral provocation test. $^{85}$

\section{Automation of the Basophil Activation Test}

Presently, the BAT is regarded as a good ex vivo test that holds many diagnostic advantages in FA which include 
high sensitivity/specificity, low risk profile, potential to predict symptom severity and importantly the potential to discriminate between sensitized asymptomatic and truly allergic patients; ${ }^{17,86}$ nevertheless, as mentioned before, there are still some hurdles to fully embrace the capabilities of this test broadly in the clinical practice.

It is not only the lack of standardization that is currently a challenge, there are also issues concerning some of the procedural limitations such as the needed flow cytometry expertise, cost, technical operational and maintenance aspects, the challenging pre-processing and prelabelling of samples and the ponderous of existing workflows. Difficulties like this mean that currently the BAT remains limited to specialized laboratories.

The BAT is performed in a limited number of laboratories as blood sampling and storage for this type of test requires special conditions to preserve cell viability and functionality (eg, sample needs to remain refrigerated) and should be used within 8 hours after obtaining the sample, although some researchers currently suggest it can be as long as 24 hours, ${ }^{87,88}$ since at room temperature IgE mediated reactions decrease faster. ${ }^{59}$ Currently, there are several time limits mentioned in the literature so there is no real consensus and this poses a problem because presently the inclination is to obtain the sample and perform the test so quickly that it is a challenge. ${ }^{15}$

In order to reduce hands-on time, efforts have been made to develop a simplified and standardized wholeblood based BAT prototype procedure to increase the automation of this test. Arif-Lusson et al proposed a whole blood based and simplified procedure for the BAT which relies on a dry antibody formulation technology that can be transposed in a 96 well plate format. ${ }^{89}$ Moreover, a novel microfluidic-based immunoaffinity BAT (miBAT) has been introduced to simplify the cumbersome BAT processes and therefore makes it more accessible to clinical practice. This microfluidic device is coated with anti-CD203c and designed to capture basophils from whole blood directly which are then activated by anti-FceRI antibody and followed by optical detection of CD63 expression. ${ }^{90}$ Blood collected from allergic patients and healthy controls was analyzed with the miBAT with the expression of CD63 percentage significantly higher after allergen activation when compared to the negative control $(\mathrm{p}<0.001)$ and miBAT data were comparable to flow cytometry. ${ }^{91}$ This technique however still needs validation in larger patient populations to assess its performance.
In addition to the storage time of the sample, there is a lack of consensus regarding which of the existing BAT protocols should be applied to harmonize results and therefore be able to compare them between laboratories. Behrends et al recently published a simplified protocol with automation of sample preparation, measurement and data analysis plus lengthening of the time between blood collection and sample processing. ${ }^{86}$ The researchers created a novel gating strategy with 3 antibodies (FceRI $\alpha, C D 203 c$, CD63) which was compared to their previous protocol that used 12 antibodies; the results found no differences in sensitivity and specificity between the two protocols or between the automated and the manually analysed samples, which saved $90 \%$ of labor time. Moreover, this new protocol by Behrends considerably extended the time frame for performing a BAT after blood donation to 7 days for whole blood storage at room temperature and 17 days at $4^{\circ} \mathrm{C}$ prior to BAT preparation and measurement. The researchers confirmed their results via a nationwide ring trial that showed a robust and applicable BAT protocol in a variety of flow cytometers. ${ }^{86}$ Agyemang et al took a different approach to preserve basophil activation stability and therefore expand the use of the BAT. ${ }^{92}$ Agyemang et al evaluated a novel peanut-BAT (P-BAT) as a diagnostic method of peanut allergy; in this pilot study basophils in whole blood were stimulated with six peanut concentrations $(0.0001-10 \mathrm{mg} /$ $\mathrm{mL}$ ) within 3 to 4 hours of sample acquisition and activation was measured by $\mathrm{CD} 63+$ and $\mathrm{CD} 203 \mathrm{c}+$ expression via flow cytometry on days $0,1,3$ and 5. Findings showed basophil activation at each peanut concentration with the P-BAT method on day 0 , and it was sustained on days 1,3 and 5 without further stimulation; therefore by using the P-BAT method researchers eliminated the immediate need for sample processing by a simple activating basophils in a simple way using whole blood, which can be easily performed in the first 4 hours of sample collection. ${ }^{92}$

\section{Passive BAT}

Directly exposing basophils from fresh whole blood or isolated peripheral blood mononuclear cells to various allergen concentrations is a technique known as "direct BAT". ${ }^{14}$ This technique has certain limitations; for example, the recommendation that the test has to be performed within 8 to 24 hours after collection of the blood sample as basophil reactivity decreases over time ${ }^{88}$ and a key problem known as "anergy", which means non-responding basophils to $\operatorname{IgE}$ receptormediated signaling after stimulation with one or more types of allergen or anti- FceRI IgE receptor stimulation, a problem 
that presents in about $10 \%$ of the individuals. ${ }^{15}$ This "basophil anergy" appears to be associated with a down-regulation of basophil Syk expression and function plus an apparent reduction in the incidence of allergic rhinitis. ${ }^{93}$

Research has shown that it is possible to reproduce passive sensitization of basophils with IgE ex vivo, this technology can be applied to allergic diseases as well. In the "passive" (also called "indirect") BAT (iBAT) isolated basophils from pooled healthy donor blood are stripped from receptor bound $\operatorname{IgE}$ and passively sensitized with sIgE from an allergic patient's serum. Subsequently, these basophils can be activated by exposing them to the allergens of interest. This allows the storage of large amounts of patient serum and to determine the allergic status at once at a later point in time, solving one of the crucial performance issues with the BAT. ${ }^{14}$ Moreover, by using pooled blood from several donors the risk for "basophil anergy" is reduced. This technique demands more time and is more labor intensive when compared to the direct BAT, but the advantages it presents for researchers and clinicians for exploring immunological allergy mechanisms as well as optimizing allergy diagnosis and monitoring treatment efficacy should not be overlooked. ${ }^{14}$ A different approach to combat the issue of nonresponding basophils was taken by Santos et al, by researching if the ability to elicit peanut-induced cell activation could be transferred by passive sensitization of LAD2 mast cells with patients plasma. ${ }^{84}$ The mast cell activation test (MAT) to peanut strongly correlated with the BAT $\left(R_{S}=0.808, \mathrm{P}<0.001\right)$ plus the MAT gave a conclusive results for participants with non-responding basophils; however, the BAT showed a greater diagnostic accuracy due to its higher sensitivity. ${ }^{94}$
Additionally it is important to note that by using a passive BAT the influence of the patient basophils are not taken into account which might be an important factor. However, a study regarding peanut allergy and the use of the $\mathrm{iBAT}$ predicted a peanut allergy which is comparable to studies that used the direct BAT. ${ }^{17}$ Nevertheless, it is unknown whether intrinsic basophil characteristics can play a minor role in some patients.

\section{Conclusions}

The current challenge in FA diagnosis is to develop a technique which is both accessible and reliable plus could replace the expensive, time-consuming and patient-unfriendly OFCs. The key messages from this review are summarized in Figure 2. Currently, the BAT is a promising ex vivo diagnostic tool in food allergy diagnosis. Nonetheless, several methodological aspects need to be investigated before a standardized protocol is available which can be universally applied making comparisons between results of different studies justified, which are summarized in Table 1. Besides the fact that the diagnostic accuracy of the reference test, an OFC, is crucial the type of allergen used in the BAT is a main point to consider as the BAT does not reflect food processing and digestion. In addition, it is also highly necessary to standardize the basophil identification and activation markers plus procedures for detection of activation basophils. In our opinion, the way of conquering these challenges might be possible by collaboration between clinicians and laboratory technicians in multi-center studies. Furthermore, the role and applicability of current diagnostic tools, such as OFC, should be discussed in order to be able to determine the diagnostic power and place of the BAT in the current diagnostic work-up for a food allergy.

\begin{tabular}{|c|c|}
\hline Current Consensus & Possible Diagnostic Advantages \\
\hline $\begin{array}{l}\text { - The BAT is a promising ex vivo approach in food allergy } \\
\text { diagnosis, especially in special populations (e.g. young } \\
\text { children) where traditional test can yield unreliable results. } \\
\text { - The BAT has recorded high sensitivity and specificity levels; } \\
\text { however no standardization and harmonization remain an } \\
\text { obstacle for its widespread use. }\end{array}$ & $\begin{array}{l}\text { - The BAT is a surrogate parameter than can measure } \\
\text { the clinical relevance of sensitization. } \\
\text { - Current research suggests the BAT is more reliable } \\
\text { than SPT and slgE levels in differentiating food allergy } \\
\text { from sensitization in allergic children. }\end{array}$ \\
\hline $\begin{array}{l}\text { - OFC is the current gold standard for food allergy diagnosis } \\
\text { therefore it should be the best tool to validate the BAT. } \\
\text { - Several questions remain regarding the OFC (e.g. } \\
\text { reproducibility threshold value, false negative and dubious } \\
\text { outcomes), therefore caution is needed to avoid a flawed } \\
\text { validation process. }\end{array}$ & $\begin{array}{l}\text { - The BAT is particularly useful for IgE mediated food } \\
\text { allergies that tend to subside or even disappear over } \\
\text { time (such as: cow's milk and egg) by closely reflecting } \\
\text { the clinical phenotype of this type of patient. } \\
\text { - The BAT seems useful for measuring the success of } \\
\text { OIT but to what is extent is yet to be determined }\end{array}$ \\
\hline Future Validation & Useful tool in FA and OIT \\
\hline
\end{tabular}

Figure 2 Summary of the key messages of the current status of applying BAT in allergy diagnostics and immunotherapy. 


\section{Disclosure}

The authors report no conflicts of interest in this work.

\section{References}

1. National Academies of Sciences, Engineering and Medicine; Health and Medicine Division; Food and Nutrition Board. Finding a Path to Safety in Food Allergy: Assessment of Food of the Global Burden, Causes, Prevention and Management, and Public Policy. US: National Academies Press; November 30, 2016.

2. European Commission Directive. 2007/68/EC of 27TH November; 2007.

3. Gupta RS, Warren CM, Smith BM, et al. Prevalence and severity of food allergies among US adults. JAMA Netw Open. 2019;2(1): e185630. doi:10.1001/jamanetworkopen.2018.5630

4. Nwaru BI, Hicktein L, Panesar SS, Roberts G. Prevalence of common food allergies in Europe: a systematic review. Allergy. 2014;69:992-1007. doi:10.1111/all.12423

5. Mori F, Barni S, Saretta F, et al. Epidemiology of rare allergic diseases in children. Pediatr Allergy Immunol. 2020;31(26):39-42. doi:10.1111/pai.13359

6. Loh W, Tang MLK. The epidemiology of food allergy in the global context. Int J Environ Res Public Health. 2018;15:2043. doi:10.3390/ ijerph15092043

7. Kelleher MM, Jay N, Perkin MR, et al. An algorithm for diagnosing IgE-mediated food allergy in study participants who do not undergo food challenge. Clin Exp Allergy. 2020;50(3):330-342. doi:10.1111/ cea. 13577

8. Foong RX, Santos AF. Biomarkers of diagnosis and resolution of food allergy. Pediatr Allergy Immunol. 2021;32:223-233.

9. De Martinis M, Sirufo MM, Suppa M, Ginaldi L. New perspective in food allergy. Int $J$ Mol Sci. 2020;21:1474. doi:10.3390/ijms 21041474

10. King RM, Knibb RC, Hourihane JO. Impact of peanut allergy on quality of life, stress and anxiety in the family. Allergy. 2009;64 (3):461-468. doi:10.1111/j.1398-9995.2008.01843.x

11. Bird JA, Crain M, Varshney P. Food allergen panel testing often results in misdiagnosis of food allergy. $J$ Pediatr. 2015;166 (1):97-100. doi:10.1016/j.jpeds.2014.07.062

12. Santos AF, Douiri A, Becares N, et al. Basophil activation test discriminates between allergy and tolerance in peanut-sensitized children. J Allergy Clin Immunol. 2014;134(3):645-652. doi:10.10 16/j.jaci.2014.04.039

13. Ebo DG, Bridts CH, Mertens CH, Sabato V. Principles, potential, and limitations of ex vivo basophil activation by flow cytometry in allergology: a narrative review. J Allergy Clin Immunol. 2021;147 (4):1143-1153. doi:10.1016/j.jaci.2020.10.027

14. Hemmings O, Kwok M, McKendry R, Santos AF. Basophil activation test: old and new applications in allergy. Curr Allergy Asthma Rep. 2018;18(12):77. doi:10.1007/s11882-018-0831-5

15. Anostegui IJ, Melioli G, Canonica GW, et al. IgE allergy diagnostics and other relevant test in allergy, a World Allergy Organization position paper. World Allergy Organ J. 2020;13:100080. doi:10.10 16/j.waojou.2019.100080

16. Patil SU, Bunyavanich S, Phil M, Berin MC. Emerging food allergy biomarkers. J Allergy Clin Immunol Pract. 2020;8(8):2516-2524. doi:10.1016/j.jaip.2020.04.054

17. Santos AF, Shreffler WG. Road map for the clinical application of the basophil activation test in food allergy. Clin Exp Allergy. 2017; 47:1115-1124. doi:10.1111/cea.12964

18. Santos AF, James LK, Bahnson HT, et al. IgG4 inhibits peanut-induced basophil and mast cell activation in peanut-tolerant children sensitized to peanut major allergens. J Allergy Clin Immunol. 2015;135(5):1249-1256. doi:10.1016/j.jaci.2015.01.012
19. Cottel N, Saf S, Bourgoin M, et al. Two different composite markers predict severity and threshold dose in peanut allergy. J Allergy Clin Immunol Pract. 2020;9(1):275-282. doi:10.1016/j.jaip.2020.09.043

20. Savage J, Sicherer S, Wood R. The natural history of food allergy. J Allergy Clin Immunol Pract. 2016;4:196-203. doi:10.1016/j. jaip.2015.11.024

21. Niggemann B. Pitfalls in double-blind, placebo-control oral food challenges. Allergy. 2007;62:729-732. doi:10.1111/j.1398-9995.2007. 01396.x

22. Santos A, Gideon L. Basophil activation test: food challenge in a test tube or specialist research tool? Clin Transl Allergy. 2016;6(1):1-9. doi:10.1186/s13601-016-0098-7

23. Paranjape A, Tsai M, Mukai K, et al. Oral immunotherapy and basophil mast cell reactivity in food allergy. Front Immunol. 2020;11:602660. doi:10.3389/fimmu.2020.602660

24. McGowan EC. Update on the performance and application of basophil activation test. Curr Allergy Asthma Rep. 2013;13(1):101-109. doi:10.1007/s11882-012-0324-x

25. Ezendam J, van Loveren H. Parameters needed to estimate the global burden of peanut allergy. RIVM Report 340007002; 2012.

26. Vereda A, van Hage M, Ashlstedt S, et al. Peanut allergy: clinical and immunological differences among patients from 3 different geographical regions. J Allergy Clin Immunol. 2011;127:603-607. doi:10. 1016/j.jaci.2010.09.010

27. Warren C, Lei C, Sicherer S, Scheimer R, Gupta R. Prevalence and characteristics of peanut allergy is US adults. J Allergy Clin Immunol. 2021;147(6):2263-2270.

28. Duan L, Celik A, Hoang JA, et al. Basophil activation test shows high accuracy in the diagnosis of peanut and tree nut allergy: the markers of nut allergy. Allergy. 2021;76(6):1800-1812.

29. Ehlers AM, den Hartog Jager CF, Knulst AC, Otten HG. Distinction between peanut allergy and tolerance by characterization of $\mathrm{B}$ cell receptor repertoires. Allergy. 2021;76(9):2753-2764. doi:10.1111/ all.14897

30. Santos AF, Du Toit G, O'Rourke CO, et al. Biomarkers of severity and threshold of allergic reactions during oral peanut challenges. $J$ Allergy Clin Immunol. 2020;146(2):344-355. doi:10.1016/j. jaci.2020.03.035

31. Rentzos G, Lundberg V, Lundqvist C, et al. Use of basophil activation test as a complementary diagnostic tool in the diagnosis of severe peanut allergy in adults. Clin Transl Allergy. 2015;5:22. doi:10.1186/s13601-015-0064-9

32. Santos AF, Bergmann M, Caubet JC, et al. Basophil activation test reduces oral food challenges to nut and sesame. J Allergy Clin Immunol Pract. 2021;9(5):2016-2027. doi:10.1016/j.jaip.2020.12. 039

33. Elizur A, Appel MY, Nachshon L, et al. NUT Co Reactivity ACquiring Knowledge for Elimination Recommendations (NUT CRACKER) study. Allergy. 2018;73:593-601. doi:10.1111/all.13 353

34. Worm M, Hompes S, Fiedler EM, Ilner AK, Zuberbier T, Vieths S. Impact of naïve, heat-processed and encapsulated hazelnuts on the allergic response. Clin Exp Allergy. 2009;39(1):159-166. doi:10.11 11/j.1365-2222.2008.03143.x

35. Tsai M, Mukai K, Chinthrajah RS, Nadeau KC, Galli SJ. Sustained successful peanut oral immunotherapy associated with low basophil activation and peanut-specific IgE. J Allergy Clin Immunol. 2020; 145:885-896. doi:10.1016/j.jaci.2019.10.038

36. Burks AW, Wood RA, Jones SM, et al. Sublingual immunotherapy for peanut allergy: long term follow up of a randomized multicentre trial. J Allergy Clin Immunol. 2015;135(5):1240-1248. doi:10.1016/j. jaci.2014.12.1917

37. Patil SU, Ogunniyi AO, Calatroni A, et al. Peanut oral immunotherapy transiently expands circulating Ara $\mathrm{h} 2$-specific B cells with homologous repertoire in unrelated individuals. J Allergy Clin Immunol. 2015;136(1):125-134. doi:10.1016/j.jaci.2015.03.026 
38. Patil SU, Steinbrecher J, Calatroni A, et al. Early decrease in basophil sensitivity to Ara h 2 precedes sustained unresponsiveness after peanut oral immunotherapy. J Allergy Clin Immunol. 2019;144(5):13 10-1319. doi:10.1016/j.jaci.2019.07.028

39. Schoemaker AA, Sprikkelman AB, Grimshaw KE, et al. Incidence and natural history of challenge-proven cow's milk allergy in European children - EuroPrevall birth cohort. Allergy. 2015;70 (8):963-972. doi:10.1111/all.12630

40. Crittenden RG. Cow's milk allergy: a complex disorder. J Am Coll Nutr. 2005;24 (6):582S-59S. doi:10.1080/07315724.2005.10719507

41. Fiocchi A, Brozek J, Schunemann H, et al. World Allergy Organization Diagnosis and Rationale for Action against Cow's Milk Allergy (DRACMA) guidelines. Pediatr Allergy Immunol. 2010;21(21):1-125.

42. Zeng Y, Zhang J, Dong G, et al. Assessment of cow's milk-related symptom scores in early identification of cow's milk protein in Chinese infants. BMC Pediatr. 2019;19(1):1-7. doi:10.1186/s12887019-1563-y

43. Munblit D, Perkin MR, Palmer DJ, et al. Assessment of evidence about common infant symptoms and cow's milk allergy. JAMA Pediatr. 2020;174(6):599-608. doi:10.1001/jamapediatrics.2020. 0153

44. Ciepiela O, Zwiazek J, Zawadzka-Krajewska A, Kotula M, Kulus U. Basophil activation test based on the expression of $\mathrm{Cd} 203 \mathrm{c}$ in the diagnostic of cow milk allergy in children. Eur J Med Res. 2010;15 (ii):21-26. doi:10.1186/2047-783X-15-S2-21

45. Sato S, Tachimoto H, Shukuya A, et al. Basophil activation marker CD203c is useful in the diagnosis of hen's egg and cow's milk allergies in children. Int Arch Allergy Immunol. 2010;152(1):54-61. doi:10.1159/000312126

46. Rubio A, Vivinus-Nebot M, Bourrier T, Saggio B, Albertini M, Bernard A. Benefit of the basophil activation test in deciding when to reintroduce cow's milk in allergic children. Allergy. 2010;66 (1):92-100. doi:10.1111/j.1398-9995.2010.02432.x

47. Norgaard A, Bernard H, Wal JM, et al. Allergenicity of individual cow milk protein in DBPCFC-positive milk allergic adults. $J$ Allergy Clin Immunol. 1996;97(3):237. doi:10.1016/S0091-6749(96)80436-2

48. Hoffman HJ, Santos AF, Mayorga C, et al. The clinical utility of basophil activation test in diagnosis and monitoring of allergic disease. Allergy. 2015;70:1393-1405. doi:10.1111/all.12698

49. Schocker F, Kull S, Schwager C, Behrends J, Jappe U. Individual sensitization pattern recognition to cow's milk and human milk differs for various clinical manifestations of milk allergy. Nutrients. 2019;11:1331. doi:10.3390/nu11061331

50. Ruinemans-Koerts J, Schmidt-Hieltjes Y, Jansen A, Savelkoul HFJ, Plasier A, van Setten P. The basophil activation test reduced the need for a food challenge test in children suspected of $\operatorname{IgE}$ mediated cow's milk allergy. Clin Exp Allergy. 2018;49(3):350-356. doi:10.1111/cea. 13307

51. Panjo GB, Caminiti L, Ruggeri P, et al. Oral immunotherapy for cow's milk allergy with a weekly up-dosing regimen: a randomized single-blind controlled study. Ann Allergy Asthma Immunol. 2010;24 (4):376-381.

52. Martorell A, De la Hoz B, Ibanez MD, et al. Oral desensitization as a useful treatment in 2 year old children with cow's milk allergy. Clin Exp Allergy. 2011;41(9):1297-1304. doi:10.1111/j.1365-2222.2011. 03749.x

53. Burbank AJ, Sood P, Vickery BP, Wood RA. Oral immunotherapy for food allergy. Immunol Allergy Clin North Am. 2016;36:55-69. doi:10.1016/j.iac.2015.08.007

54. Passalacqua G, Landi M, Pajno GB. Oral immunotherapy for cow's milk allergy. Curr Opin Allergy Clin Immunol. 2012;12:271-277. doi:10.1097/ACI.0b013e3283535b93

55. Nucera E, Schiavino D, D’Ambrosio C, et al. Immunological aspects of oral desensitization in food allergy. Dig Dis Sci. 2000;45:637-641. doi:10.1023/A:1005430231735
56. Nucera E, Pecora V, Buonomo A, et al. Utility of basophil activation test for monitoring the acquisition of clinical tolerance after oral desensitization to cow's milk: Pilot Study. United European Gastroenterol J. 2015;3(3):272-276. doi:10.1177/2050640615570694

57. Savage JH, Matsui EC, Skirpak JM, Wood RA. The natural history of egg allergy. J Allergy Clin Immunol. 2007;120(6):1413-1417. doi:10.1016/j.jaci.2007.09.040

58. Mather P, Pfleghaar JL. Egg Allergy. Stat Pearls Publications; 2020.

59. Ocmant A, Mulier S, Hanssens L, et al. Basophil activation test for the diagnosis of food allergy in children. Clin Exp Allergy. 2009;39 (8):1234-1245. doi:10.1111/j.1365-2222.2009.03292.x

60. Berin MC, Grishin A, Masilamani M, et al. Egg-specific IgE and basophil activation but not egg-specific T-cell counts correlate with phenotypes of clinical egg allergy. J Allergy Clin Immunol. 2018;142 (1):149-158. doi:10.1016/j.jaci.2018.01.044

61. Ibáñez MD, Escudero C, Sánchez-García S, Rodriguez Del Rio P. Comprehensive review of current knowledge on egg immunotherapy. J Investig Allergol Clin Immunol. 2015;25:316-328.

62. Gamboa PM, Garcia-Lirio E, Gonzalez C, Gonzales A, MartinezAranguren RM, Sanz Maria L. Is the quantification of antigen-specific basophil activation a useful tool for monitoring oral tolerance induction in children with egg allergy? J Investig Allergol Clin Immunol. 2016;26(1):25-30. doi:10.18176/jiaci.0004

63. Giavi S, Vissers YM, Muraro A, et al. Oral immunotherapy with low allergenic hydrolysed egg in egg allergic children. Allergy. 2016;71:1575-1584. doi:10.1111/all.12905

64. Grabenhenrich LB, Reich A, McBride D, et al. Physician's appraisal vs documented signs and symptoms in the interpretation of food challenge test: the EuroPrevall birth cohort. Pediatr Allergy Immunol. 2018;29(1):58-65. doi:10.1111/pai.12811

65. Grabenhenrich LB, Reich A, Bellach J, et al. A new framework for the documentation and interpretation of oral food challenges in population-based and clinical research. Allergy. 2017;72(3):45 3-461. doi:10.1111/all.13049

66. Van Erp FC, Knulst AC, Meijer Y, Gabriele C, van der Ent CK. Standardized food challenges are subject to variability in interpretation of clinical symptoms. Clin Transl Allergy. 2014;4:43. doi:10.1186/s13601-014-0043-6

67. Brand PL, Landzaaat-Berghuizen MA. Differences between observers in interpreting double-blind placebo-controlled food challenges: a randomized trial. Pediatr Allergy Immunol. 2014;25(8):755-759. doi:10.1111/pai.12313

68. Glaumann S, Nopp A, Johansson SGO, Borres MP, Nilsson C, Sampson AP. Oral peanut challenge identifies an allergy but the peanut allergen threshold sensitivity is not reproducible. PLoS One. 2013;8(1):e53465. doi:10.1371/journal.pone.0053465

69. Sampson HA, Gerth van Wijk R, Bindsley-Jensen C, et al. Standardizing double-blind, placebo-controlled oral food challenge: American Academy of Allergy, Asthma \& Immunology - European Academy of Allergy and Clinical Immunology PRACTALL consensus report. J Allergy Clin Immunol. 2012;130:1260-1274. doi:10.10 16/j.jaci.2012.10.017

70. Santos A, Alpan O, Hoffmann HJ. Basophil activation test: mechanisms and considerations for it use in clinical trials and clinical practice. Allergy;. 2021;76:2420-2432. doi:10.1111/all.14747

71. Schwager C, Kull S, Behrends J, et al. Peanut oleosins associated with severe peanut allergy-importance of lipophilic allergens for comprehensive allergy diagnostics. Allergy Clin Immunol. 2017;140 (5):1331-1338. doi:10.1016/j.jaci.2017.02.020

72. Jappe U, Schwager C. Relevance of lipophilic allergens in food allergy diagnosis. Curr Allergy Asthma Rep. 2017;17(9):61. doi:10.1007/s11882-017-0731-0

73. Vissers YM, Iwan M, Adel-Patient K, et al. Effect of roasting on the allergenicity of major peanut allergens Ara h 1 and Ara h 2/6: the necessity of degranulation assays. Clin Exp Allergy. 2011;41 (1):1631-1642. doi:10.1111/j.1365-2222.2011.03830.x 
74. Sabato V, van Hegel AJ, de Knop KJ, et al. Basophil activation reveals divergent patient-specific responses to thermally processed peanuts. J Investig Allergol Clin Immunol. 2011;21(7):527-531.

75. Sancho AI, Rigby NM, Zuidmeer L, et al. The effect of thermal processing on the $\mathrm{IgE}$ reactivity of the non-specific lipid transfer protein from apple, Mal d 3. Allergy. 2005;60(10):1262-1268. doi:10.1111/j.1398-9995.2005.00876.x

76. Eberlein B, Hann R, Eyerich S, et al. Optimizing of the basophil activation test: comparison of different basophil identification markers. Cytometry B Clin Cytom. 2015;88(3):183-189. doi:10. 1002/cytob. 21203

77. Depince-Berger AE, Sidi-Yahya K, Jeraibt M, Lambert C. Basophil activation test: implementation and standardization between systems and between instruments. J Quant Cell Sci. 2017;91(3):261-269.

78. Kim T, Yu J, Li H, et al. Validation of inducible basophil biomarkers: time, temperature and transportation. Clin Cytom. 2021. doi:10.1002/ cyto.b. 21991

79. Lotzch B, Dolle S, Vieths S, Worm M. Exploratory analysis of CD63 and CD203c expression in basophils from hazelnut sensitized and allergic individuals. Clin Transl Allergy. 2016;6:45. doi:10.1186/ s13601-016-0134-7

80. Zenarruzabeitia O, Vitalle J, Orrantia A, et al. CD300c costimulates IgE-mediated basophil activation and its expression is increased in patients with cow milk's allergy. J Allergy Clin Immunol. 2019;143 (2):700-711. doi:10.1016/j.jaci.2018.05.022

81. Sabato V, Verweij MM, Bridts $\mathrm{CH}$, et al. CD300a is expressed on human basophils and seems to inhibit IgE/FceRI dependent anaphylactic degranulation. Cytometry B Clin Cytom. 2012;82B:132-138. doi:10.1002/cyto.b.21003

82. Clark GJ, Ju X, Tate C, Hart DN. The CD300 family of molecules are evolutionary significant regulators of leucocyte functions. Trends Immunol. 2009;30:209-217. doi:10.1016/j.it.2009.02.003

83. Patil SU, Calatroni A, Schneider M, et al. Data driven programmatic approach to analysis of basophil activation tests. Cytometry B Clin Cytom. 2018;94(4):667-673. doi:10.1002/cyto.b.21537

84. Santos AF, Du Toit G, Douiri A, et al. Distinct parameters of the basophil activation test reflect the severity and threshold of allergic reactions to peanut. J Allergy Clin Immunol. 2015;135(1):179-186. doi:10.1016/j.jaci.2014.09.001
85. Mehlich J, Fischer J, Hilger C, et al. The basophil activation test differentiates between patients with alpha-gal syndrome and asymptomatic alpha-gal sensitization. J Allergy Clin Immunol. 2019;143 (1):182-189. doi:10.1016/j.jaci.2018.06.049

86. Behrends J, Schwager C, Hein M, Scholzen T, Kull S, Jappe U. Innovative robust basophil activation test using a novel gating strategy reliably diagnosing allergy with full automation. Allergy. 2021:1-13. doi:10.1111/all.14900

87. Eberlein B. Basophil activation as marker of clinically relevant allergy and therapy outcome. Front Immunol. 2020;11:1815. doi:10.3389/fimmu.2020.01815

88. Mukai K, Gaudenzio N, Gupta S, et al. Assessing basophil activation by using flow cytometry and mass cytometry in blood stored 24 hours before analysis. J Allergy Clin Immunol. 2017;139(3):889-899. doi:10.1016/j.jaci.2016.04.060

89. Arif-Lusson R, Agabriel C, Carsin A, et al. Streaming basophil activation testing to enable assay miniaturization and automation of sample preparation. J Immunol Methods. 2020;481-482:112793.

90. Aljadi Z, Kalm F, Ramachandraiah H, Nopp A, Lundahl J, Russomi A. Microfluidic immunoaffinity basophil activation test for point-of-care allergy diagnosis. J Appl Med. 2019;4(2):152-163. doi:10.1373/jalm.2018.026641

91. Aljadi Z, Kalm F, Nilsson C, et al. A novel tool for clinical diagnosis of allergy operating a microfluidic immunoaffinity basophil activation test technique. Clin Immunol. 2019;209:108268. doi:10.1016/j. clim.2019.108268

92. Agyemang A, Suprun M, Suarez-Farinas M, et al. A novel approach to the basophil activation test for characterizing peanut allergic patients in the clinical setting. Allergy. 2021;76(7):2257-2259. doi:10.1111/all.14752

93. Puan KJ, Andianppan AK, Lee B, et al. Systematic characterization of basophil anergy. Allergy. 2017;72(3):373-384. doi:10.1111/all.12 952

94. Santos AF, Cuoto-Francisco N, Becares N, Kwok M, Bahnson HT, Lack G. A novel human mast cell activation test for peanut allergy. J Allergy Clin Immunol. 2018;142(2):689-691. doi:10.1016/j.jaci.20 18.03.011

\section{Publish your work in this journal}

The Journal of Asthma and Allergy is an international, peer-reviewed open-access journal publishing original research, reports, editorials and commentaries on the following topics: Asthma; Pulmonary physiology; Asthma related clinical health; Clinical immunology and the immunological basis of disease; Pharmacological interventions and new therapies. The manuscript management system is completely online and includes a very quick and fair peer-review system, which is all easy to use. Visit http://www.dovepress.com/testimonials.php to read real quotes from published authors. 